\title{
Myocardial Bridge Anatomical Characteristics Relationship with ECG Changes and Markers
}

\author{
Hussein Nafakhi ${ }^{1{ }^{*}}$, Abdulameer A. Al-Mosawi ${ }^{2}$, Samet Kasim ${ }^{1}$ \\ ${ }^{1}$ Internal Medicine Department, Medicine College, University of Kufa, Najaf, Iraq \\ ${ }^{2}$ Radiology Department, Medicine College, University of Kufa, Najaf, Iraq
}

\section{ARTICLE INFO}

Article History

Received 12 December 2019

Accepted 08 April 2020

Keywords

Myocardial bridge

ECG

coronary atherosclerosis

$\mathrm{CT}$ angiography

\begin{abstract}
Background: The clinical significance of coronary Myocardial Bridge (MB) anatomical characteristics is controversial in the literature. It is not clear whether MB has a benign or malignant clinical outcome.

Objectives: To investigate the possible relationship between MB characteristics (depth, length and volume index) with ECG markers [T Peak-to-End interval (Tp-e), transmural dispersion of repolarization and Index of Cardiac Electrical Balance (iCEB)] and changes (ST-T and S/R ratio changes in V1 and aVL leads).

Patients and Methods: Forty-one patients who were diagnosed as having MB at multi-detector CT exam were enrolled in the study.

Results: MB depth and volume index were associated with notched QRS ( $p=0.001$ and 0.003 respectively) in the whole sample. The association of notched QRS with MB depth and volume index was more significant in patients with coronary atherosclerosis ( $p=0.001$ and 0.01 respectively). There was a statistically significant association between MB length $(p=0.006)$ and volume index $(p=0.001)$ with an increased $\mathrm{S} / \mathrm{R}$ ratio in aVL lead in patients free from coronary atherosclerosis. No statistically significant association was observed between MB anatomical characteristics with ECG markers of increased arrhythmia $(p>0.01)$, including TDR, Tp-e and iCEB.
\end{abstract}

Conclusion: $\mathrm{MB}$ depth and volume index showed a statistically significant association with notched QRS, particularly in patients with coronary atherosclerosis.

\section{HIGHLIGHTS}

- The clinical significance of coronary Myocardial Bridge (MB) anatomical characteristics is controversial in the literature.

- It is not clear whether MB has benign or malignant clinical outcome.

- The results of the present study showed that some MB anatomical characteristics are associated with significant ECG changes.

(C) 2020 Association for Research into Arterial Structure and Physiology. Publishing services by Atlantis Press International B.V. This is an open access article distributed under the CC BY-NC 4.0 license (http://creativecommons.org/licenses/by-nc/4.0/).

\section{INTRODUCTION}

Myocardial Bridge (MB) is a congenital coronary artery variant occurs when a part of the epicardial coronary artery, most commonly seen in the Left Anterior Descending Artery (LADA) and less frequently seen in the Right Coronary Artery (RCA) and Left Circumflex (LCx) artery, covered by myocardial fibers for a variable distance. $\mathrm{MB}$ is traditionally regarded as an incidental finding and may remain clinically silent in the majority of patients [1-3].

However, recent clinical case reports and observational studies have suggested that MB may be able to induce coronary ischemia, cardiac arrhythmia and sudden cardiac death in apparently healthy

"Corresponding author.Email: husseinaf.alnaffakh@uokufa.edu.iq

Peer review under responsibility of the Association for Research into Arterial Structure and Physiology

Data availability statement: The data that support the findings of this study are available from the corresponding author $(\mathrm{HN})$, upon reasonable request. individuals without cardiac risk factors of atherosclerosis. The proposed mechanisms for these adverse cardiac events caused by MB presence including systolic compression, spasm, plaque and premature atherosclerosis development proximal and distal to the bridge and endothelial dysfunction $[1,3]$.

It has been postulated that the anatomic characteristics of MB such as site, depth length and volume index (MB depth multiplied by MB length) may be related to coronary ischemia and the occurrence of adverse cardiac events even in patients exhibit no considerable coronary atherosclerosis. However, it is still not clear how the $\mathrm{MB}$ anatomical characteristics modulate the occurrence of adverse events or interfere with therapeutic strategies $[4,5]$.

Recently, a new emerging ECG markers, in particular Transmural Dispersion of Repolarization (TDR), defined by the T Peakto-End (Tp-e) interval and $\mathrm{Tp}-\mathrm{e} / \mathrm{QT}$, and Index of Cardiac Electrophysiological Balance (iCEB), defined by QT/QRS, and notching of QRS are reported to be accepted markers of increased 
risk or ventricular arrhythmia and sudden cardiac death in coronary artery disease [6,7].

Recently, TDR, Tp-e/QT, iCEB and notching of QRS have been suggested to be associated with MB presence $[2,6,7]$.

ECG changes, including S/R ratio and non-specific ST and T-wave abnormalities specifically at V1 and aVL leads have been suggested to be associated with LADA lesions responsible for overt coronary disease and cardiac remodeling even in the absence of symptomatic coronary artery disease $[1,2,4]$.

However, the exact role of these ECG changes or markers to the development of adverse cardiac events in patients with $\mathrm{MB}$ and their association with $\mathrm{MB}$ anatomical properties is obscured and not yet fully studied in the literature.

The main aim of this study was to investigate the possible relationships between MB characteristics (depth, length, volume and site) with ECG markers and changes.

\section{PATIENTS AND METHODS}

This cross-sectional study was carried out at the Cardiology Center at Al-Sader Teaching city between January 2016 and December 2018.

Patients recruited in this study were consecutive patients with suspected coronary artery disease who underwent 64-slice Multidetector CT (MDCT) angiography examination to exclude coronary artery disease. Forty-one patients who were diagnosed as having MB at MDCT exam were enrolled in the study.

Medical history of conventional cardiac risk factors for coronary atherosclerosis was obtained from each patient through a questionnaire at the time of coronary MDCT angiography examination including a positive family history of premature coronary artery disease (occurring before the age of 55 years in men and before 65 years in women), current smoking history (more than 10 cigarettes per day in the last year), a history of hypertension or use of antihypertension medications, hyperlipidemia (defined as levels of total cholesterol $\geq 200 \mathrm{mg} / \mathrm{dl}$ or triglyceride $\geq 150 \mathrm{mg} / \mathrm{dl}$ ) or use of lipid-lowering drugs, a history of diabetes mellitus or use of insulin or diabetes lowering drugs and measurement of body weight and height to calculate Body Mass Index (BMI) [2].

Verbal informed consent was obtained from all participants before enrolment. The study was approved by our Medicine College Board.

\subsection{MDCT Scan Protocol}

CT coronary angiography was performed with a 64-slice scanner (Aquilon 64, v. 4.51 ER 010; Toshiba Medical Systems, Tochigi, Japan). The MDCT data analyses were assessed as per our previous study [2].

\subsubsection{MB length, depth and volume index}

The length of the MB was defined as the distance of the bridged artery from the entrance to the exit beneath the myocardial band. The depth of the MB was defined as the thickness of the deepest part from the surface of the overlying myocardial fibers to the bridged segment, on the cross-sectional images. The MB volume index was defined as MB depth (in $\mathrm{mm}$ ) multiplied by MB length (in $\mathrm{mm}$ ) [4].

The analysis of MDCT images were performed by two experienced radiologists in the coronary MDCT angiography data analysis.

\subsubsection{ECG examination}

The 12-lead ECGs were recorded in the 12-lead format at a paper speed of $25 \mathrm{~mm} / \mathrm{s}$ and voltage of $10 \mathrm{~mm} / \mathrm{mV}$ by using a standard ECG system (Marquette Electronics, Milwaukee, WI, USA) while the patient was resting in the supine position. ECG readings measured manually by two cardiologists blinded to MDCT results, using calipers and a magnifying glass. R- and S-wave amplitudes were assessed to calculate $\mathrm{S} / \mathrm{R}$ ratio in V1 and aVL leads. QRS duration in ms calculated from the end of PR to the end of S-wave. The Tp-e interval calculated from the peak of the T-wave to the end of the T-wave in the precordial leads. The QT interval calculated from the beginning of the QRS complex to the end of the T. The mean value of the measurements was used in the analysis [2].

The Tp-e/QT, which represents TDR and QT/QRS, which represents the $\mathrm{iCEB}$ were calculated from these measurements. Interobserver variability was $<10 \%$.

With the regard to ECG changes, ST-segment deviation defined as an elevation or depression of $>0.1 \mathrm{mV}$ at $80 \mathrm{~ms}$ after the $\mathrm{J}$ point in all leads. T-wave inversion defined as $\mathrm{T}$-wave $>1 \mathrm{~mm}$ below $\mathrm{TP}$ segment in all leads. Diffuse ST-T changes defined when $\geq 2$ contagious and noncontiguous leads showed ST depression or elevation or T inversion [2].

Notched QRS defined if there was an end-QRS notch or a slur on the downslope of a prominent R-wave [6].

\subsection{Statistical Analysis}

Data are expressed as mean \pm standard deviation or as number\% or as median (interquartile range), as appropriate. The associations between MB length, depth and volume index with continuous ECG variables examined using Spearman's rank correlation. Student's $t$-test was used to compare MB length, depth and volume index among dichotomous ECG variables, including T-wave inversion and notched QRS. The multiple regression analysis was not performed due to the small sample size. A $p$-value $\leq 0.01$ was used to indicate statistical significance to decrease the risk of randomly generated significant results by multiple testing. SPSS ver. 23.0 (SPSS Inc., Chicago, IL, USA) was used for statistical analysis.

\section{RESULTS}

Forty-one patients with MB (age $56 \pm 10$ years, $56 \%$ male) were enrolled in the study. There were no ST-segment depression or elevation reported no MB patients in the present study. Patients' characteristics are shown in Table 1.

The statistical analysis was performed in two steps:

First, we studied the relationship of MB characteristics with ECG markers and changes in the whole sample (41 patients with $\mathrm{MB}$ ). 
Table 1 Patient's characteristics

\begin{tabular}{|c|c|}
\hline Parameters & $\begin{array}{c}\text { Mean } \pm \text { SD or } n \% \\
\text { or median (IQR) }\end{array}$ \\
\hline Age & $56 \pm 10$ \\
\hline Male & $56 \%$ \\
\hline Hypertension & $54 \%$ \\
\hline Diabetes mellitus & $20 \%$ \\
\hline Dyslipidemia & $36 \%$ \\
\hline Family history & $10 \%$ \\
\hline Smoking & $27 \%$ \\
\hline BMI & $29 \pm 5$ \\
\hline \multicolumn{2}{|l|}{ MB characteristics } \\
\hline Length $(\mathrm{mm})$ & $21 \pm 9$ \\
\hline Depth (mm) & $3.5 \pm 1.7$ \\
\hline Volume index (mm) & $82 \pm 22$ \\
\hline \multicolumn{2}{|l|}{ MB site } \\
\hline LADA MB & $93.5 \%$ \\
\hline RCA MB & $5 \%$ \\
\hline LCx MB & $2.5 \%$ \\
\hline CAC & $0(0-54)$ \\
\hline \multicolumn{2}{|l|}{ ECG markers } \\
\hline QRS (ms) & $77 \pm 22$ \\
\hline QT (ms) & $397 \pm 61$ \\
\hline Tp-e (ms) & $73 \pm 12$ \\
\hline iCEB & $5.6 \pm 2$ \\
\hline TDR & $0.18 \pm 0.0$ \\
\hline Notched QRS & $15 \%$ \\
\hline Diffuse $\mathrm{T}$ inversion & $27 \%$ \\
\hline $\mathrm{T}$ inversion $\mathrm{V} 1$ & $41 \%$ \\
\hline $\mathrm{S} / \mathrm{R}$ aVL & $0.2 \pm 0.5$ \\
\hline S/R V1 & $5 \pm 1.9$ \\
\hline
\end{tabular}

Second, we classified the MB patients into two groups according to Coronary Artery Calcium (CAC) score values: patients without coronary atherosclerosis or normal coronary arteries $(\mathrm{CAC}=0)$ group (19 patients) and patients with coronary atherosclerosis (CAC >0) group (22 patients). The relationship of MB characteristics with ECG changes and markers were analyzed in each group separately.

\subsection{MB Length Relationship with ECG Markers and Changes}

Myocardial bridge length mean was $21 \pm 9 \mathrm{~mm}$. There was no statistically significant association between MB length with ECG changes and markers $(p>0.01)$ in the whole sample, as in Table 2.

After dividing patients into two groups according to CAC score values, a statistically significant association was observed between MB length with an increased S/R ratio in aVL lead $(p=0.006)$ in the patients without coronary atherosclerosis, while no statistically significant association was observed between MB length with other ECG markers and changes $(p>0.01)$, in both coronary atherosclerosis and without coronary atherosclerosis groups, as in Tables 3 and 4.

\subsection{MB Depth Relationships with ECG Markers and Changes}

Myocardial bridge depth mean was $3.5 \pm 1.7 \mathrm{~mm}$. A statistically significant association was observed between $\mathrm{MB}$ depth with notched
Table 2 Association of MB characteristics with ECG changes and markers in the whole sample

\begin{tabular}{|c|c|c|c|c|c|c|}
\hline \multirow{2}{*}{ ECG markers } & \multicolumn{2}{|c|}{ MB length } & \multicolumn{2}{|c|}{ MB depth } & \multicolumn{2}{|c|}{ MB volume } \\
\hline & $r$ & $p$-value & $r$ & $p$-value & $r$ & $p$-value \\
\hline QRS & 0.1 & 0.3 & 0.3 & 0.02 & 0.3 & 0.03 \\
\hline QT & 0.0 & 0.5 & 0.1 & 0.4 & 0.0 & 0.6 \\
\hline Tp-e & 0.2 & 0.1 & 0.0 & 0.6 & 0.1 & 0.2 \\
\hline iCEB & 0.0 & 0.5 & 0.2 & 0.1 & 0.2 & 0.1 \\
\hline TDR & 0.2 & 0.1 & 0.1 & 0.3 & 0.2 & 0.1 \\
\hline $\mathrm{T}$ inversion $\mathrm{V} 1$ & - & 0.2 & - & 0.06 & _- & 0.1 \\
\hline Diffuse $\mathrm{T}$ inversion & - & 0.5 & _- & 0.6 & _- & 0.8 \\
\hline Notched QRS & - & 0.1 & - & 0.001 & - & 0.003 \\
\hline $\mathrm{S} / \mathrm{R} \mathrm{aVL}$ & 0.3 & 0.02 & 0.0 & 0.6 & 0.2 & 0.1 \\
\hline S/R V1 & 0.0 & 0.5 & 0.0 & 0.8 & 0.0 & 0.8 \\
\hline
\end{tabular}

Table 3 Association of MB characteristics with ECG changes and markers in patients without coronary atherosclerosis

\begin{tabular}{|c|c|c|c|c|c|c|}
\hline \multirow{2}{*}{ ECG markers } & \multicolumn{2}{|c|}{ MB length } & \multicolumn{2}{|c|}{ MB depth } & \multicolumn{2}{|c|}{ MB volume } \\
\hline & $r$ & $p$-value & $r$ & $p$-value & $r$ & $p$-value \\
\hline QRS & 0.0 & 0.9 & 0.1 & 0.6 & 0.1 & 0.5 \\
\hline QT & 0.0 & 0.8 & 0.2 & 0.2 & 0.1 & 0.5 \\
\hline Tp-e & 0.0 & 0.9 & 0.1 & 0.4 & 0.0 & 0.8 \\
\hline iCEB & 0.0 & 0.9 & 0.0 & 0.9 & 0.0 & 0.8 \\
\hline TDR & 0.1 & 0.6 & 0.0 & 0.8 & 0.1 & 0.6 \\
\hline $\mathrm{T}$ inversion $\mathrm{V} 1$ & _- & 0.3 & - & 0.06 & - & 0.1 \\
\hline Diffuse $\mathrm{T}$ inversion & - & 0.3 & - & 0.3 & - & 0.2 \\
\hline Notched QRS & - & 0.02 & - & 0.03 & 0.6 & 0.003 \\
\hline S/R aVL & 0.6 & 0.006 & 0.5 & 0.03 & 0.7 & 0.001 \\
\hline S/R V1 & 0.2 & 0.2 & 0.2 & 0.2 & 0.2 & 0.2 \\
\hline
\end{tabular}

Table 4 Association of MB characteristics with ECG changes and markers in patients with coronary atherosclerosis

\begin{tabular}{|c|c|c|c|c|c|c|}
\hline \multirow{2}{*}{ ECG markers } & \multicolumn{2}{|c|}{ MB length } & \multicolumn{2}{|c|}{ MB depth } & \multicolumn{2}{|c|}{ MB volume } \\
\hline & $r$ & $p$-value & $r$ & $p$-value & $r$ & $p$-value \\
\hline QRS & 0.3 & 0.1 & 0.5 & 0.01 & 0.5 & 0.005 \\
\hline QT & 0.1 & 0.3 & 0.1 & 0.6 & 0.1 & 0.6 \\
\hline Tp-e & 0.4 & 0.04 & 0.4 & 0.05 & 0.4 & 0.04 \\
\hline ICEB & 0.2 & 0.3 & 0.3 & 0.09 & 0.3 & 0.07 \\
\hline TDR & 0.3 & 0.1 & 0.3 & 0.09 & 0.4 & 0.05 \\
\hline $\mathrm{T}$ inversion $\mathrm{V} 1$ & - & 0.8 & - & 0.5 & - & 0.6 \\
\hline Diffuse $\mathrm{T}$ inversion & - & 0.9 & - & 0.3 & - & 0.6 \\
\hline Notched QRS & - & 0.4 & - & 0.001 & - & 0.01 \\
\hline $\mathrm{S} / \mathrm{R}$ aVL & 0.2 & 0.2 & 0.2 & 0.2 & 0.2 & 0.2 \\
\hline S/R V1 & 0.2 & 0.2 & 0.2 & 0.2 & 0.2 & 0.2 \\
\hline
\end{tabular}

QRS ( $p=0.001)$. In contrast, there was no significant association between MB depth with other ECG changes and markers in the whole sample, as in Table 2.

In patients without coronary atherosclerosis, a statistically significant association was observed between MB depth with notched QRS $(p=0.001)$ in the coronary atherosclerosis group, while no statistically significant association was observed between $\mathrm{MB}$ depth with other ECG markers and changes $(p>0.01)$, in both coronary atherosclerosis and without coronary atherosclerosis groups, as in Tables 3 and 4. 


\subsection{MB Volume Index Relationships with ECG Markers and Change}

A statistically significant association was observed between $\mathrm{MB}$ volume index with notched QRS $(p=0.003)$ in the whole sample, as in Table 2. A statistically significant association was observed between $\mathrm{MB}$ volume index with increased $\mathrm{S} / \mathrm{R}$ ratio in aVL lead $(p=0.001)$ and notched QRS $(p=0.003)$ in patients without coronary atherosclerosis, while no statistically significant association was observed between MB volume index with other ECG markers and changes $(p>0.01)$, in both coronary atherosclerosis and without coronary atherosclerosis groups, as in Tables 3 and 4.

\section{DISCUSSION}

The main results of the present study are as follows: (1) MB depth and volume index were associated with notched QRS complex; (2) a statistically significant association of MB length and volume index with increased S/R ratio in aVL lead was observed in patients without coronary atherosclerosis.

Myocardial bridge is considered to be the most common congenital abnormality encountered in coronary computed tomographic studies. However, the exact prevalence of coronary MB and its cardiovascular consequences varies widely among studies according to the method used for the detection of $\mathrm{MB}$ presence and selection of enrolled patients $[2,3]$.

Emerging data suggest that specific anatomic characteristics of myocardial bridges, such as length, depth and volume index, may be contributed to the development of atherosclerosis and related to subsequent adverse cardiac events and increased morbidity [3,7].

It was believed that long and deep MB leads to an augmentation of the systolic compression of the involved coronary artery causing an increase of retrograde blood flow toward the proximal segment and disturbance of blood hemodynamics to the peripheral myocardium [8].

Moreover, $\mathrm{MB}$ volume index was significantly larger in patients with coronary atherosclerosis than that in cases without cardiac lesions [9].

Recently, fragmentation or notching of QRS complex is proposed to be a novel and simple ECG marker that can be used in identifying patients at high risk for arrhythmic events $[6,10]$.

Seo et al. [6] reported that the notched type of QRS was more frequently observed in MB involving LADA.

Also, early repolarization abnormalities and terminal notching of QRS were reported in young patients with chest pain and MB of LADA, but without occlusive coronary atherosclerosis, with perfusion defects matched the areas supplied by the bridged part of the LADA in the absence of occlusive coronary atherosclerosis [11].

It has been suggested that subtle ECG changes and repolarization abnormalities in patients with MB may precede the development of occlusive coronary atherosclerosis [12].

Generally, MB patients showed nonspecific ECG changes of coronary ischemia or conduction abnormalities in the resting state $[1,2]$.
It has been suggested that isolated ST-T changes at V1 and aVL leads are associated with lesions in the left anterior descending artery $[2,13]$.

Patients with MB may have dynamic precordial ST-T changes, mimicking Wellen's ECG pattern, and repolarization disturbances secondary to myocardial edema rather than left ventricular systolic dysfunction in the absence of occlusive coronary retry disease [14].

The association of MB length and volume index with increased $\mathrm{S} / \mathrm{R}$ ratio in aVL lead in patients with normal coronary arteries, but not in patients with coronary atherosclerosis, in the present study raises the question about the subtle non-ischemic ECG changes in patients with $\mathrm{MB}$ with subsequent changes secondary to the effect of progressive coronary atherosclerosis process.

In the present study, no statistically significant association was observed between MB anatomical characteristics with ECG markers of increased arrhythmia $(p>0.01)$, including TDR, Tp-e and iCEB.

It has been reported that a hemodynamically significant MB morphological features are associated with increased myocardial fibrosis and edema, both of which are linked with an increased risk of electrical instability of the heart [15].

However, a positive association between MB presence and ECG markers of increased risk of sudden death and malignant arrhythmia has not been yet definitely proved, despite many small studies and case reports support this potential association $[15,16]$.

In agreement with our findings, Ylldırım et al. [16] showed no significant association between MB length with ECG repolarization markers, including TDE and Tp-e interval.

The clinical relevance of the association of $\mathrm{MB}$ with long-term adverse outcomes and increased risk of sudden cardiac death secondary to electrical changes related to $\mathrm{MB}$ anatomical properties was debated among previous case reports. Several case reports showed a possible link between $\mathrm{MB}$ with an increased risk of sudden death and adverse clinical outcome [15-18].

On the other hand, other case reports showed that a good longterm outcome for patients with MB involving LADA [2,9].

These debating results regarding the association of $\mathrm{MB}$ with increased risk of cardiac mortality and morbidity reflecting the heterogeneous nature of $\mathrm{MB}$ presence and that not all myocardial bridges are the same with different hemodynamic and cardiac consequences related to specific $\mathrm{MB}$ anatomical characteristics, particularly MB length and depth.

There are several limitations to this study. First, controlling for all possible factors or residual confounding that might affect ECG markers was difficult. Second, the small number of study patients did not allow assessment of the association of MB site with ECG markers and changes or performing regression analysis, which may limit the generalizability of our results. Third, the lack of an appropriate control group to compare the results as patients without $\mathrm{MB}$ have no anatomical characteristics to be compared with ECG findings. A prospective study with a larger number of individuals with $\mathrm{MB}$ is required to confirm the results of the present study and its clinical significance.

The results of the present study may provide a valuable insight, which could be used in large-scale, follow-up studies for better 
understanding of the prognostic and clinical significance of $\mathrm{MB}$ anatomical characteristics.

In conclusion, $\mathrm{MB}$ depth and volume index showed a statistically significant association with notched QRS, particularly in patients with coronary atherosclerosis. The clinical significance of the present findings needs to be validated in a larger prospective study.

\section{CONFLICTS OF INTEREST}

The authors declare they have no conflicts of interest.

\section{AUTHORS' CONTRIBUTION}

HN and AAA study conceptualization and writing (review and editing) the manuscript. HN and SK Data curation, formal analysis and writing (original draft). AAA project administration. AAA and $\mathrm{HN}$ supervised the project.

\section{FUNDING}

There were no external funding sources for this study.

\section{ACKNOWLEDGMENTS}

The authors acknowledge Dr. Hasan Alnafakh for his valuable contribution to the analysis of MDCT images in the present study.

\section{REFERENCES}

[1] Rovai D, Di Bella G, Pingitore A, Coceani M. Myocardial bridging: a review with emphasis on electrocardiographic findings. Ann Noninvasive Electrocardiol 2015;20:103-7.

[2] Nafakhi H, Al-Mosawi AA, Hassan MB, Hameed F, Alareedh $\mathrm{M}, \mathrm{Al}$-Shokry W. ECG changes and markers of increased risk of arrhythmia in patients with myocardial bridge. J Electrocardiol 2019;56:90-3.

[3] Nakanishi R, Rajani R, Ishikawa Y, Ishii T, Berman DS. Myocardial bridging on coronary CTA: an innocent bystander or a culprit in myocardial infarction? J Cardiovasc Comput Tomogr 2012;6:3-13.

[4] Ishikawa Y, Akasaka Y, Suzuki K, Fujiwara M, Ogawa T, Yamazaki $\mathrm{K}$, et al. Anatomic properties of myocardial bridge predisposing to myocardial infarction. Circulation 2009;120:376-83.
[5] Ishii T, Ishikawa Y, Akasaka Y. Myocardial bridge as a structure of "double-edged sword" for the coronary artery. Ann Vasc Dis 2014;7:99-108.

[6] Seo J, Park J, Oh J, Uhm JS, Sung JH, Kim JY, et al. High prevalence and clinical implication of myocardial bridging in patients with early repolarization. Yonsei Med J 2017;58:67-74.

[7] Brodsky SV, Roh L, Ashar K, Braun A, Ramaswamy G. Myocardial bridging of coronary arteries: a risk factor for myocardial fibrosis? Int J Cardiol 2008;124:391-2.

[8] Ishikawa Y, Kawawa Y, Kohda E, Shimada K, Ishii T. Significance of the anatomical properties of a myocardial bridge in coronary heart disease. Circ J 2011;75:1559-66.

[9] Yukio I, Yoko K, Ehiichi K, Toshiharu I. (1) coronary events caused by myocardial bridge. Ann Vasc Dis 2009;2:79-94.

[10] Cetin MS, Ozcan Cetin EH, Canpolat U, Cay S, Topaloglu S, Temizhan A, et al. Usefulness of fragmented QRS complex to predict arrhythmic events and cardiovascular mortality in patients with noncompaction cardiomyopathy. Am J Cardiol 2016;117:1516-23.

[11] Deveci M, Babaoğlu K, Kayabey Ö. A rare cause of early repolarisation in an adolescent boy with chest pain: myocardial bridging. Cardiovasc J Afr 2017;28:e5-e7.

[12] Hostiuc S, Rusu MC, Hostiuc M, Negoi RI, Negoi I. Cardiovascular consequences of myocardial bridging: a meta-analysis and meta-regression. Sci Rep 2017;7:14644.

[13] Ak R, Doganay F, Onur OO, Akoglu EU. Electrocardiographic clue for a mid-LAD lesion. BMJ Case Rep 2016;2016:pii: bcr2015213046.

[14] Migliore F, Zorzi A, Marra MP, Basso C, Corbetti F, De Lazzari $\mathrm{M}$, et al. Myocardial edema underlies dynamic T-wave inversion (Wellens' ECG pattern) in patients with reversible left ventricular dysfunction. Heart Rhythm 2011;8:1629-34.

[15] Hostiuc S, Curca GC, Dermengiu D, Dermengiu S, Hostiuc M, Rusu MC. Morphological changes associated with hemodynamically significant myocardial bridges in sudden cardiac death. Thorac Cardiovasc Surg 2011;59:406-10.

[16] Yıldırım E, İpek E, Kalkan K, Öztürk M, Cengiz M, Hamur H. The relationship between myocardial bridge and electrocardiographic Tp-e interval, Tp-e/QT and Tp-e/QTc ratio. Med Bull Haseki 2017;55:21-6.

[17] Nguyen TH, Burnside PR, Dieter RS, Nanjundappa A. Right coronary artery distribution of myocardial bridging: an unusual case presenting with ST-elevation myocardial infarction. Tex Heart Inst J 2007;34:489-91.

[18] Riezzo I, Monciotti F, Pomara C, Fineschi V. Myocardial bridging of the right coronary artery and emotional stress: a fatal link? Int J Cardiol 2007;115:e99-e101. 